\title{
FEM model-based optimal control synthesis for curing a large composite structure with CAD imported geometry
}

\author{
Sergey Shevtsov ${ }^{1, a}$, Ilya Tarasov², Vladimir Axenov ${ }^{3}$, Igor Zhilyaev ${ }^{1}$, Jiing-Kae Wu ${ }^{4}$ and Natalia Snezhina ${ }^{5}$ \\ ${ }^{1}$ Southern Center of Russian Academy, Aircraft Technologies Department, 344006 Rostov on Don, Russia \\ ${ }^{2}$ AP Group LLC, Department of New Materials, 192236 Saint Petersburg, Russia \\ ${ }^{3}$ LANIT Group, CAD Department, 129075 Moscow, Russia \\ ${ }^{4}$ National Kaohsiung Marine University, Mechanical Engineering Department, 81157 Kaohsiung city, Taiwan R. O.C. \\ ${ }^{5}$ Don State Technical University, Aircraft Engineering Department, 344000 Rostov on Don, Russia
}

\begin{abstract}
The paper studies a problem of optimal control synthesis for curing a large composite structure using open die moulding in an autoclave. The necessary control should eliminate early hard skin formation, emergence of resin-rich or resin-dry areas, insufficient consolidation, and uneven cure. This purpose is achieved through providing uniform distribution of degree of cure and temperature within the cured part volume. The used approach is illustrated on the example of the large CFRP aircraft panel manufactured by means of two-stage curing cycle. The forward problem combines the heat transfer and autocatalytic kinetic equations, which are linked by the specific heat capacity, thermal conductivity coefficient and by the exothermal heat source that depend on the prepreg's degree of cure. The control synthesis problem is formulated as a multi-objective optimization problem where minimized objectives are deviations of temperature and degree of cure within a cured part, considering constraints imposed by thermal-kinetic properties of prepreg and manufacturing requirements. The Pareto-based optimization algorithm, which executes the mapping of a subset of design space to the objectives space by the cyclic calling the forward problem implemented as a finite element model, allows estimating the best achievable quality indicators of manufactured composite parts, finding satisfactory parameters of the control law, and decision-making considering all imposed constraints.
\end{abstract}

\section{Introduction}

The landscape of composite part manufacturing has been spreading rapidly over the recent years. The growth in use of composites is the most intensive in aircraft and rotorcraft industries due to increased specific and fatigue strength or stiffness and better weight saving of these materials, which significantly exceed the same properties of metal lightweight alloys. Most typical polymeric composites reinforced with glass / carbon fibers with the thermoset resin matrix are used in manufacturing of such parts as wing and tail skins, spars, stringers, ribs, fuselage sections, helicopters main and tail rotor blades, pressure vessels, radomes, cowlings, etc. [1].

Components based on long fibers, tows, yarn, or fabrics are manufactured using some kind of laminating procedure. In this process, sheets of reinforcement precoated with resin (prepreg) or freshly applied resin are forced against the surface of a mould under required conditions of pressure, temperature, and time. Many different technologies have been developed to produce aircraft components of different shape, dimensions, loadcarrying, and wall thickness. A wide variety of components that have the open-shell geometry or smooth panel shape are widespread in aircraft structures. The open-mould forming is most suitable for components with such types of geometry. According to this technology involving the use of only one mould surface, over which layers of reinforcing fibres or fabric are tightly placed, the laminate with orthotropic or transversely isotropic structural symmetry is most often formed. Then a mould packed with a raw material is placed into a vacuum bag, which is a flexible plastic film embracing the surface of the lay-up, and a vacuumtight bag together with a mould is disposed in an autoclave $[1,2]$.

The autoclave system includes a vessel containing a bag mould with laid-up prepreg, sources for heating the gas stream and circulating it uniformly inside the vessel, a subsystem for pressurizing the gas stream, a subsystem for

a Corresponding author: sergnshevtsov@gmail.com 
applying vacuum to the part covered with a vacuum bag, and a subsystem for controlling operating parameters. Gas circulation inside an autoclave is essential to provide mass flow for temperature uniformity and heat transfer to the part load. The vacuum is applied to remove air and volatiles from the polymerized prepreg, while the pressure is required to consolidate all layers together into a laminate. At the first stage of heating the prepreg, resin viscosity in prepreg plies decreases, attains a minimum, and then increases rapidly (gelation stage) as the curing (cross-linking) reaction begins and proceeds toward completion. The temperature up to $150^{\circ} \mathrm{C}-200^{\circ} \mathrm{C}$ may be required to complete the polymerization process of epoxy resin systems is most often applied to the open mould using hot-air blowers and electric elements installed in an autoclave [1, 2]. Figure 1 shows a typical two-stage cure cycle for a glass / carbon fiber-epoxy prepreg.

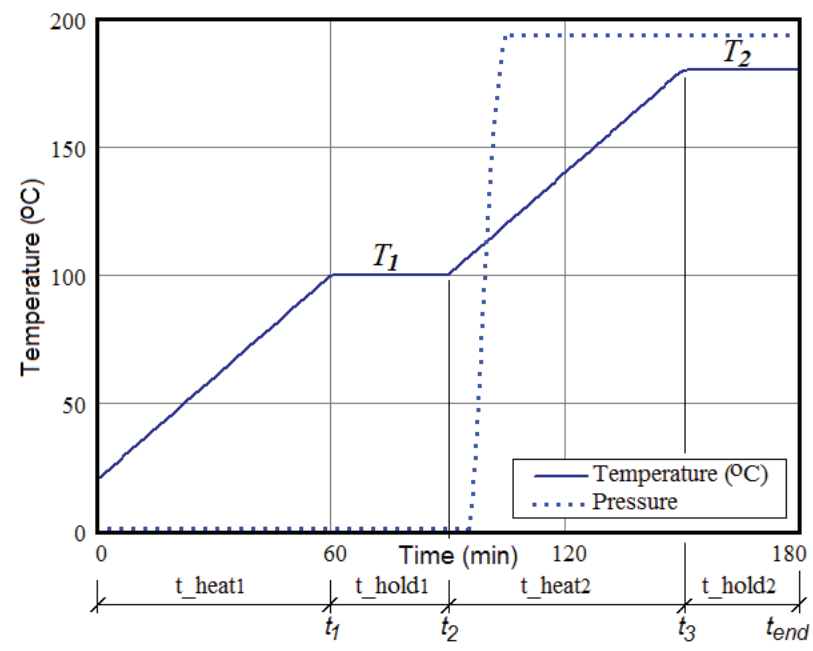

Figure 1. An example of two-stage cure cycle for a carbon fiber-epoxy prepreg.

The first stage of this cure cycle consists of increasing the temperature at a controlled rate up to the start of resin fluidization and dwelling at this temperature until the minimum resin viscosity is reached. Such an isothermal hold is applied at the stages of resin fluidization as well as at the process completion to allow the temperature distribution to become more uniform, especially in large components with thickness variations, to prolong the time allowed for volatiles removal, and to allow pre-reactions and consolidation of the resin. At the end of cure cycle after resin vitrification, the temperature is slowly reduced while the laminate is still under pressure. The ready part is removed from the mould and, sometimes, postcured at an elevated temperature.

In order to eliminate problems such as overheating, early hard skin formation (which prevents the release of gases), porosity, resin-rich areas, resin-dry areas, poor surface finish, insufficient consolidation, and uneven cure, the correct timing of applications of temperature and pressure should be used. All means of process monitoring that support traditional autoclave curing can provide information on temperature, pressure, and sometimes resin viscosity (with DEA sensors placed in some points of the cured part). Unfortunately, these means cannot indicate the state of cure throughout the cured component [3]. Due to this circumstance, the composite technology most often uses the trial-and-error approach to the tooling design and process development that causes the high cost of advanced composites. Such a difficulty is most significant for the large composite structures and can be overcome by using methods of process modelling or simulation that can predict a desired tooling and process design window, within which a reduced number of trials can be conducted [1].

A good process model should correctly describe a number of coupled physical and chemical phenomena, including heat transfer, thermal, kinetic and rheological properties of material transformation at all stages of cure, and should take into account different constraints imposed by the process equipment, material properties, etc. This requires preliminary resin testing using DSC, DMA, DEA techniques to identify all resin properties during the full cure cycle. Due to the complex mathematical description of the problem and complexity of the components geometry, the finite element method is the most efficient and has been used recently by many authors to model a variety of polymeric composite cure processes [4 - 7]. Such a finite element implementation of the cure process allows formulating an optimization problem where the degree of cure at the final stage of the curing process and variations of the degree of cure and temperature within the cured body during the whole process can be accepted as objectives, whereas time dependencies of applied heat flow, temperature and pressure are design variables. Such an approach, which has been implemented in some recent works [8 - 15], allows synthesizing the most appropriate process control that provides the best quality of the cured part [16 - 18] for the acceptable processing time as well as determining some process parameters, which are related to the state of the controlled plant and can be effectively monitored, e.g., temperature at some accessible points on the mould or cured part surface. The multi-objective approach to the problem optimization of such a system is the most efficient in a practice when mapping of the area in the design space to the objective space is considered, and a pseudo optimal solution is selected, taking into account various constraints, including trade-offs between the full processing time, maximum allowable temperature gradient and curing uniformity. 
Such a multi-objective optimization model based approach to the synthesis of the pseudo optimal process control is illustrated in this paper using an example of the cure process of carbon fiber reinforced epoxy-based large composite panel with many longitudinally oriented reinforcing ribs (see Fig. 2 ). The part with open-shell geometry and wall thickness varied in the range of $5-8 \mathrm{~mm}$ should be manufactured by means of the open mould curing technology.

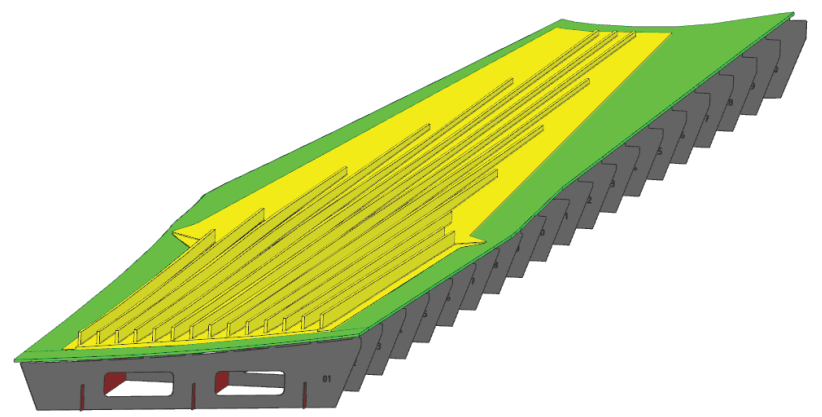

Figure 2. Geometry of the cured CFRP panel placed on the open mould, which is supported by the numerous footings that prevent the mould deformations during cure

The present study consisted of the following phases. By using the results of DSC studies we have established that the autocatalytic equation is the most appropriate to describe the resin kinetic behaviour when cured at varying heating rates. All six parameters of this equation, the value of reaction enthalpy, and heat conduction dependence on degree of cure have been identified using numerical technique of evolutionary computing [19]. DEA testing results of the prepreg that describe the transformation of the resin rheology during cure have been analyzed to determine the temperatures of both isothermal holds, which are present in Fig.1.

The geometry of the cured part together with a mould made of thick-walled CFRP has been redesigned to exclude some topological imperfection, and exported to the finite element soft package. The governing system of coupled heat transfer and kinetic equations for the prepreg is similar to that used in [15], but it has different boundary conditions and dependencies of thermal, kinetic and rheological properties on the temperature and degree of cure. Assuming a two-stage cure cycle, we varied the duration of the first and second heating up and two dwelling sections to provide minimum variations of the degree of cure and temperature gradient within the cured part, while maximum duration of each cure stage and the whole cure cycle and temperatures at the first and second isothermal holds are constrained. Optimum area in a four-dimensional design space has been visualized by the set of its two-dimensional projections with the contour lines that determine the values of the objectives.

\section{Identification of cure kinetics and prepreg properties}

In order to determine the thermal and kinetic properties of the used prepreg Toray T800, all dynamic DSC tests have been carried out using NETZSCH equipment at the heating rates $0.8 \ldots 1.5 \mathrm{deg} / \mathrm{min}$. The time histories of the differential heat flow have been numerically processed to fit them by the analytical dependencies given by different kinetic models. The main criterion for choosing the most appropriate kinetic model was the ability to successfully describe the behaviour of experimental data obtained at the different heating rate. The quality indicator of these data approximation by each studied kinetic equation was the discrepancy between experimental and calculated cure rate (see Fig. 3) and was averaged on the used temperature interval. The principal types of the kinetic equations are listed in [20]. Among these types the Kamal \& Sourour equation (below simply named as Kamal)

$$
\partial \alpha / d t=A_{2} \exp \left(-E_{2} / R T\right) \cdot \alpha^{m}(1-\alpha)^{n}
$$

and autocatalytic-1 equation (below named as autocatalytic)

$$
\partial \alpha / d t=\left[A_{1} \exp \left(-\frac{E_{1}}{R T}\right)+A_{2} \exp \left(-\frac{E_{2}}{R T}\right) \cdot \alpha^{m}\right](1-\alpha)^{n},
$$

where degree of cure $\alpha$ is defined by the relationship $Q_{\text {exo }}=Q_{\text {tot }} \cdot \partial \alpha / \partial t$ between actual value of exothermal heat $Q_{\text {exo }}$ and enthalpy $Q_{t o t}$ of cure reaction; $E_{1}, E_{2}$ are the activation energies, $R$ is the universal gas constant, $T$ is the Calvin temperature, $\mathrm{m}$ and $\mathrm{n}$ are the reaction orders, and $t$ is time.
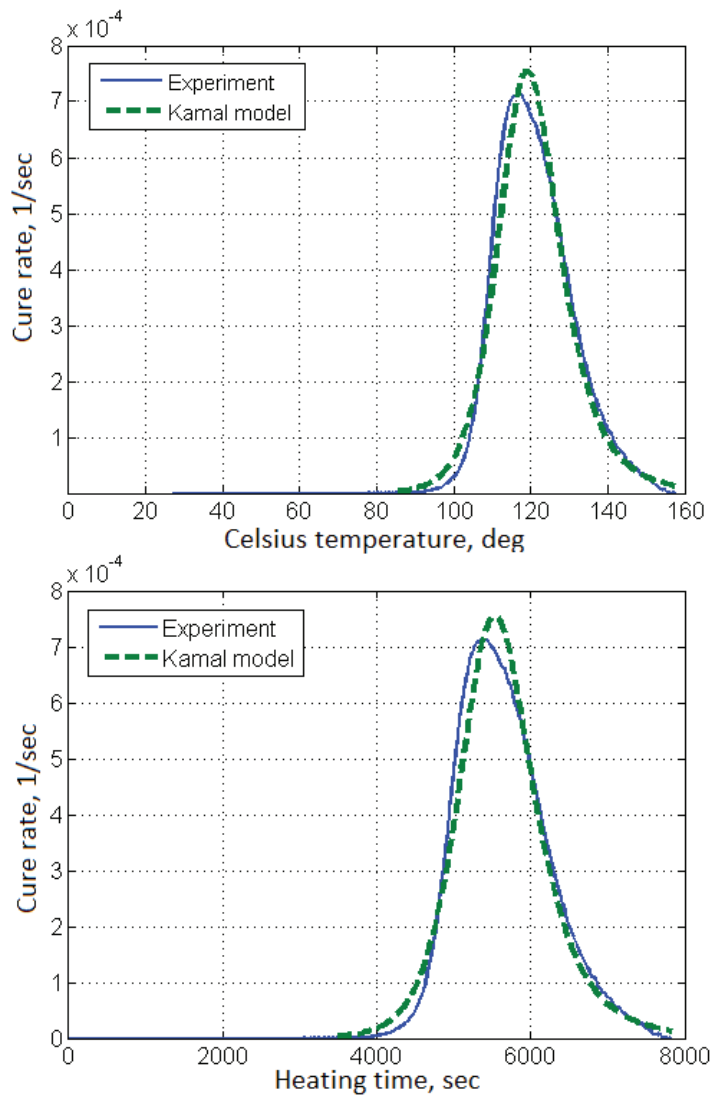
a)
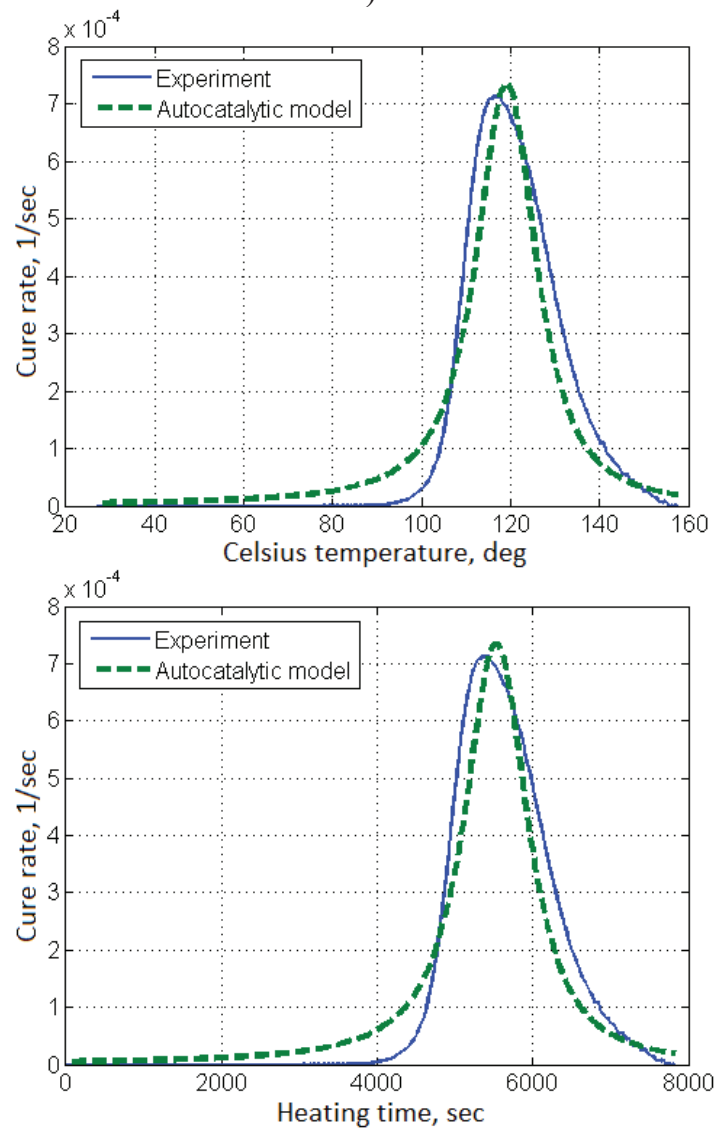

b)

Figure 3. Dependencies of cure rate obtained during the dynamic DSC test at the heating rate $1 \mathrm{deg} / \mathrm{min}$ combined with the theoretical dependencies given by the simple Kamal (a) and autocatalytic (b) models

The main difference between equations (1) and (2) is that Kamal equation can describe each polymerization process if initial value of $\alpha>0$ only, whereas autocatalytic equation (2) provides a proper description of the cure evolution even at its zero initial condition. Figure 3 demonstrates, when parameters of equations (1) and (2) are identified using the experimental data obtained from the DSC test at the identical heating rate, Kamal model gives better consistency with experimental data. But this model is valid for only one temperature law - ramp with the heating rate at which was obtained the initial DSC data that have been used for the model identification. Indeed, figure $4 a$ demonstrates an apparent dependency of the cure rate on the temperature. But this dependence is due to various heating rate. Figure $4 \mathrm{~b}$ shows the absolute overlap of three curves of the cure rates, which have been calculated from equation (1). The main inference from this careful study is that cure evolution according to Kamal model is controlled by time, instead by the temperature.
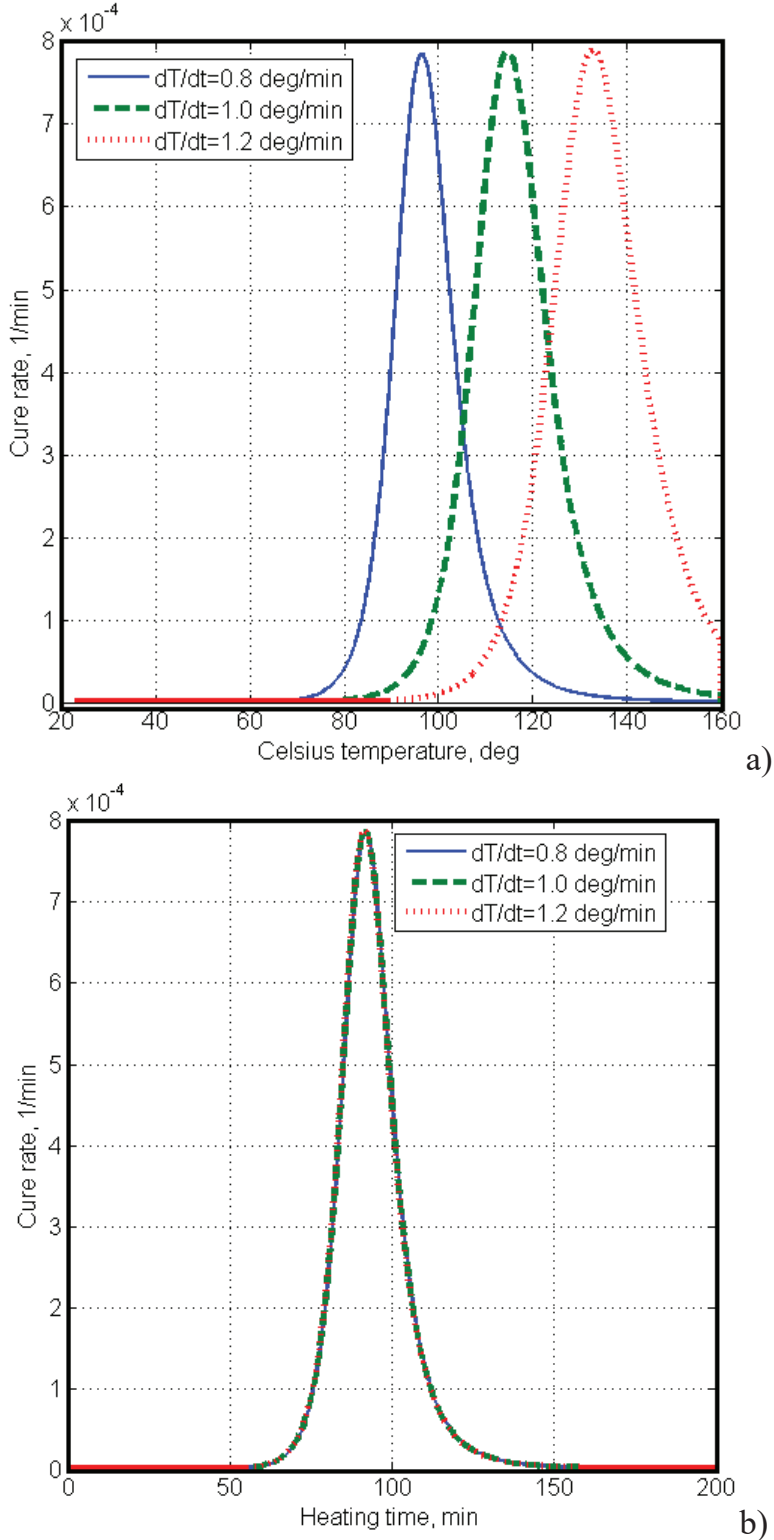

b)

Figure 4. Temperature and time dependencies of cure rate calculated from the simple Kamal equation (1) at the different heating rate

Moreover, the choice of initial value for cure in (1) is arbitrary, and it is carried out only to satisfy the requirement of better fitting the experimental data, without any reasonable justification. Meanwhile, many authors $[4,6,14-16,27]$ use this model in their studies without due justification. Hence, use the simple Kamal equation in models involving heating rate variation is improperly.

Autocatalytic model (2) doesn't have of this shortcoming. When it has been identified so, that its parameters provide the best fitting data for the dynamic DSC implemented at the boundary values of interval for the heating rate variation, this model gives the correct quantitative description of cure evolution at the heating rates inside this interval. The ability of the autocatalytic model has been confirmed in $[22,29]$ and successfully used in [11] to optimize the cure cycle of thick composite parts. Such a model has been identified and used in our 
study to formulate the coupled thermal-kinetic problem of composite part cure.

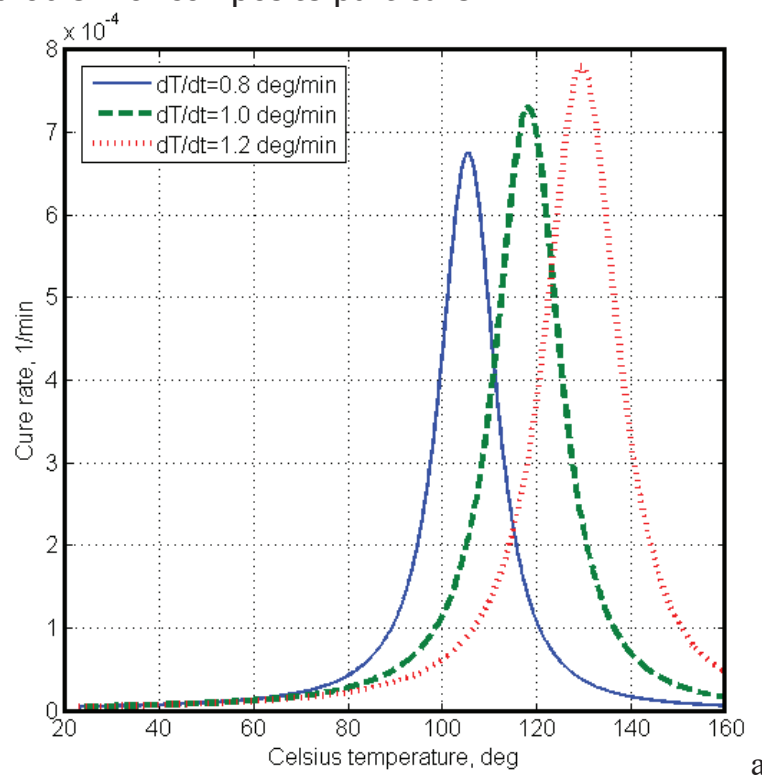

a)

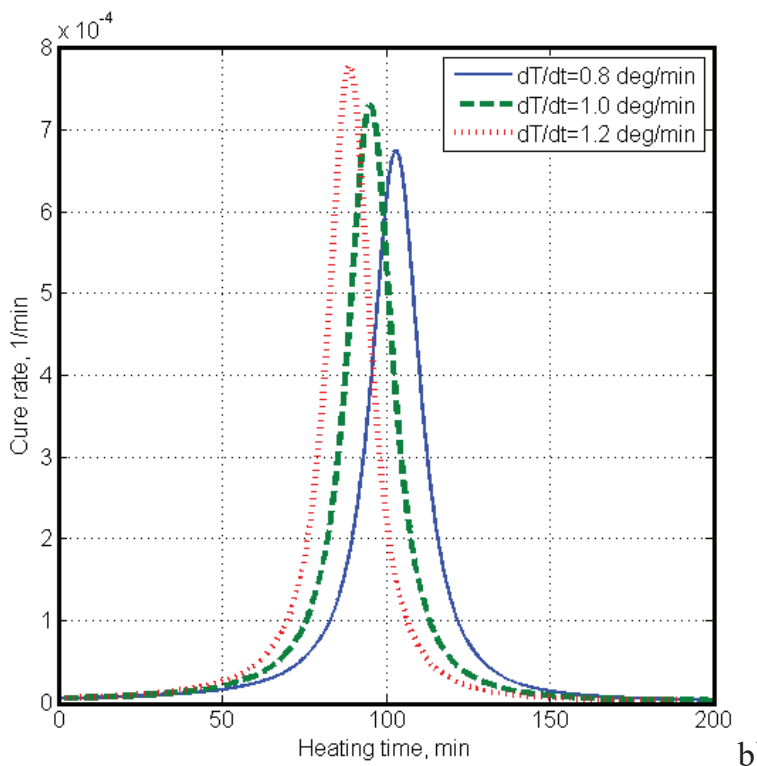

Figure 5. Temperature and time dependencies of cure rate calculated from the autocatalytic equation (2) at the different heating rate

As the further results of kinetic model identification, the enthalpy $Q_{\text {tot }}=0.105 \mathrm{MJ} / \mathrm{kg}$ of cure reaction and dependence of specific heat capacity (in $\mathrm{J} /(\mathrm{kg} \bullet \mathrm{K})$ ) on degree of cure were found

$$
C_{p}=2300-550 \cdot \alpha \text {. }
$$

\section{Rheology transformations during cure}

The rheology transformations of the prepreg during cure have been studied by DEA technique with the use NETZSCH equipment at different frequencies and varied heating laws. To prevent electrical contact of sensor Idex115 surface with conductive carbon prepreg, a special insulating layer was used to separate them. After preliminary testing, the temperature program, as present in Fig. 6, was used to determine the critical points for the cured prepreg (minimum viscosity and start of gelation inflection point).

Figures $7 \mathrm{a}$ and $7 \mathrm{~b}$ show the time and temperature dependencies of ion viscosity of the prepreg during cure and the critical points that have been used to assign the temperature of isothermal held at the finite element modelling of the part cure. Inflection points of curves coincide to the temperatures of cure rate peaks found from DSC testing. Positions of these points allow assigning instants for applying air pressure in autoclave.

\section{Forward cure modelling: problem formulation}

The full problem statement combines the heat transfer equation

$$
\rho_{c / m} C_{c / m} \partial T / \partial t+\nabla \cdot\left(-k_{c / m} \nabla T\right)=\left\{\begin{array}{l}
Q_{e x o} \\
0
\end{array}\right.
$$

with the kinetic equation (2). Subscripts $c$ and $m$ of material density $\rho_{c / m}$, specific heat capacity $C_{c / m}$, and thermal conductivities $k_{c / m}$ in equation (4) indicate the domains of composite prepreg and mould respectively. Heat sources intensity $Q_{\text {exo }}$ acts inside the composite body, while there are no any internal heat sources inside the mould. Equation (2) shows how the local value of the degree of cure depends on the temperature $T$, whose distribution is described by heat transfer equation (4). In turn, coefficients $C_{c}, k_{c}$ and internal heat sources intensity $Q_{\text {exo }}$ in equation (4) depend on the state of prepreg materials, which is a function of the degree of cure $\alpha(\mathbf{r})$. All thermal properties of the prepreg material during cure have been identified using results of experimental studies.

The initial value of the absolute temperature $T$ was taken equal to $20^{\circ} \mathrm{C}$ everywhere in simulated domains.

All surfaces of the mould and outer surfaces of the cured part were exposed to the same temperature $T(t)$ whose time history is represented in Figure 1.

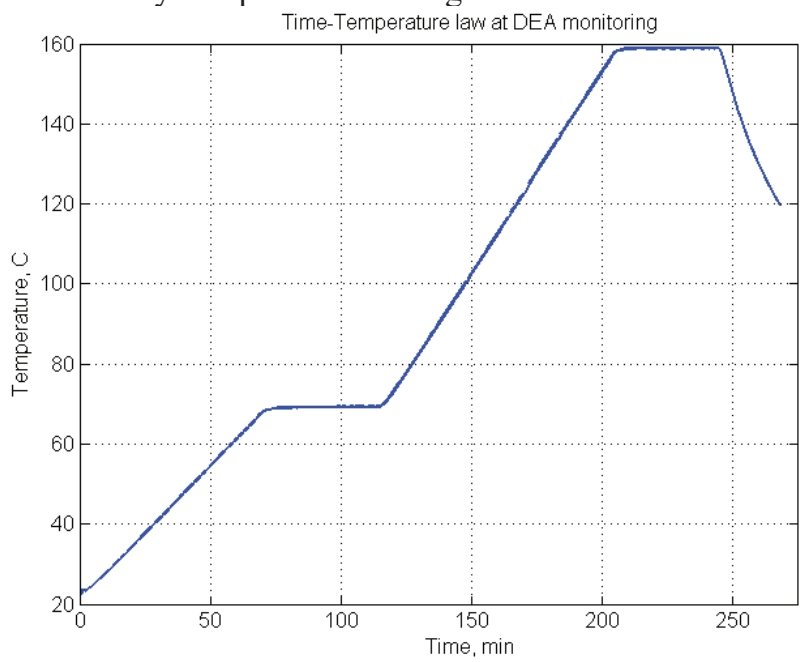

Figure 6. Temperature schedule used at DEA testing of the prepreg 


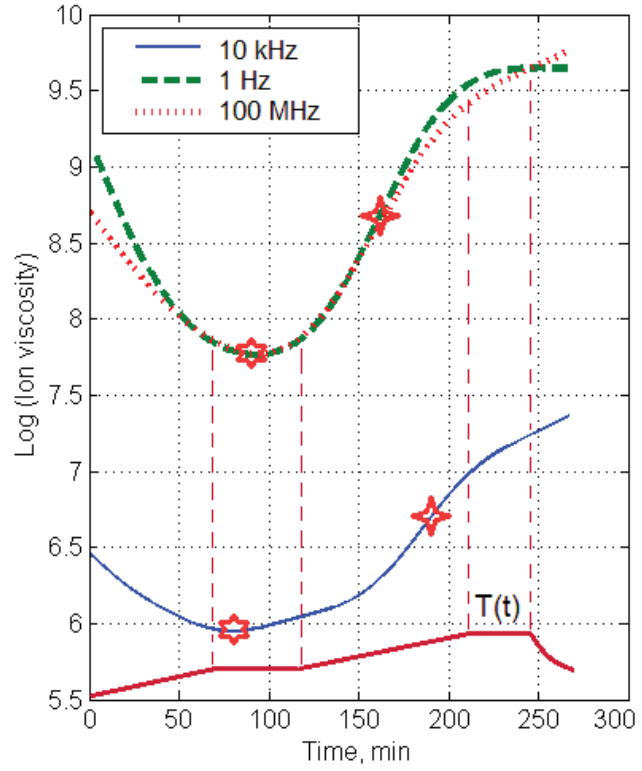

a)

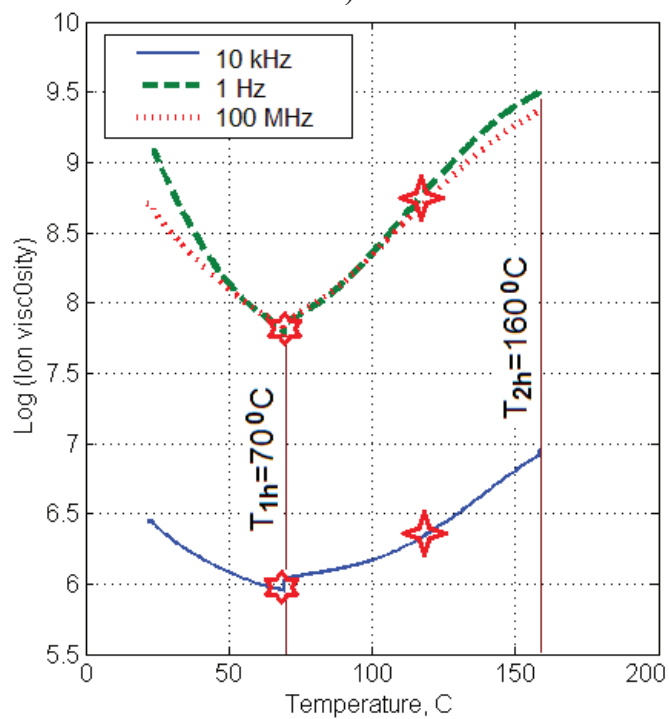

b)

Figure 7. Time (a) and temperature (b) dependencies of Log ion viscosity for the prepreg DSC testing at the heating law, which is present in Fig. 6

Four-pointed stars and hexagrams depict the critical points

To facilitate the finite element meshing the thermal resistance and thus the presence of the vacuum bag thin film were ignored, whereas release film separating prepreg and upper surface of mould was substituted by the special boundary condition

$$
\begin{aligned}
& -\mathbf{n}_{d} \cdot\left(-k\left(\nabla_{t} T_{s}+\nabla_{n} T_{s}\right)\right)=-\frac{T_{u}-T_{d}}{R_{s}}+\frac{1}{2} d_{s} \cdot Q_{s} \\
& -\mathbf{n}_{u} \cdot\left(-k\left(\nabla_{t} T_{s}+\nabla_{n} T_{s}\right)\right)=-\frac{T_{d}-T_{u}}{R_{s}}+\frac{1}{2} d_{s} \cdot Q_{s},
\end{aligned}
$$

where

$$
R_{s}=-\frac{d_{s}}{k} ; \quad T_{u}=\left.T_{s}\right|_{L=0} ; \quad T_{d}=\left.T_{s}\right|_{L=d_{s}},
$$

$\mathbf{n}_{u}$ and $\mathbf{n}_{d}$ are the normal vectors to the upper and lower boundaries of the film layer, $d_{s}$ is the film thickness, $Q_{s}=0$ is the internal heat source intensity that acts in the film (no exothermal heat) and $T_{s}$ is the auxiliary variable, which is determined inside virtual layer only.

Our numerical experiments that have been implemented with meshed thin layer (thickness $0.1 \mathrm{~mm}$ ) and virtual layer with boundary conditions (5) and (6) showed that the temperatures discrepancy in all used ranges didn't exceed $0.02{ }^{\circ} \mathrm{C}$.

The values of materials properties for CFRP mould and release film are present in Table 1.

Table 1. Material properties for mould and release film.

\begin{tabular}{|c|c|c|}
\hline Material property & CFRP mould & $\begin{array}{c}\text { Release } \\
\text { film }\end{array}$ \\
\hline Density, $\mathrm{kg} / \mathrm{m}^{3}$ & 1500 & 1200 \\
\hline $\begin{array}{c}\text { Thermal } \\
\text { conductivity, } \\
\mathrm{W} /(\mathrm{m} * \mathrm{~K})\end{array}$ & $0.75+0.1(\mathrm{~T}-20) / 180$ & 0.25 \\
\hline $\begin{array}{c}\text { Specific heat } \\
\text { capacity, } \mathrm{J} /(\mathrm{kg} * \mathrm{~K})\end{array}$ & $850+550(\mathrm{~T}-20) / 200$ & 1170 \\
\hline
\end{tabular}

In order to find an appropriate finite element meshing and indicative values of the time integration step, several segments were cut from a whole panel (see Fig. 8).

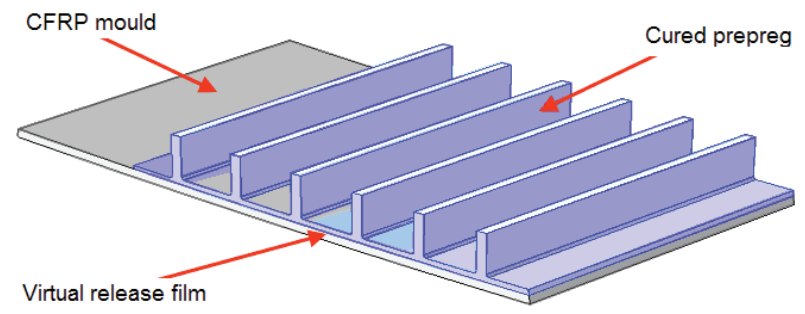

Figure 8. An example of segment that was cut from a whole panel for the preliminary testing FEM model.

All boundary conditions for these segments are illustrated in Fig. 9. The results of these preliminary simulations then have been used at the finite element implementation of forward problem of whole panel curing process in Comsol Multiphysics 5.2 environment.
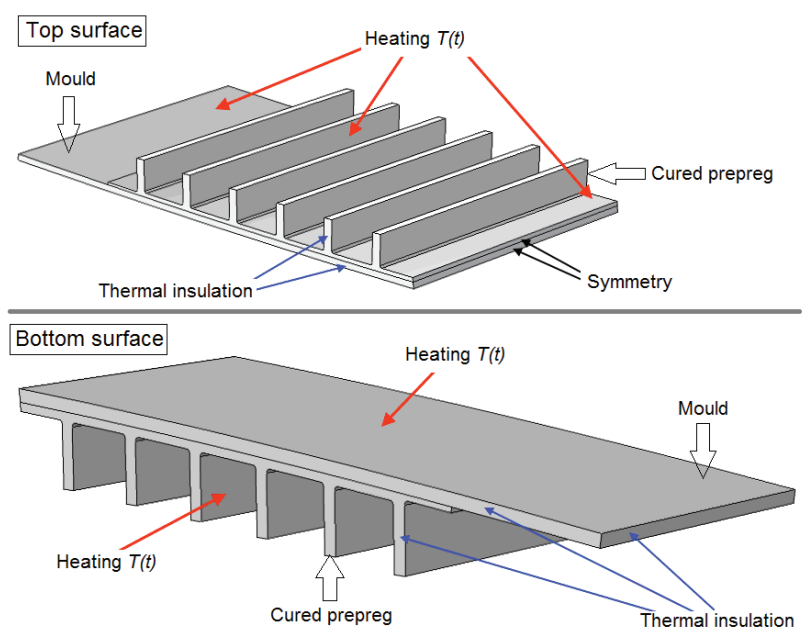
Figure 9. External boundary condition for the segments of part at the finite element transient analysis cure process

\section{Optimization problem statement and implementation}

The optimization procedure was organized as follows. The FE model of part placed onto a mould performed a parametric sweep of design variables inside pre-assigned intervals. Full cure cycle has been performed for each set of design variables, and these results were stored in text file. So, the operating algorithm fills the area in the design space with points, mapping them to the areas in the objective space. The calculation results then were numerically analyzed to satisfy all imposed constraints and reconstruct the optimum areas. Four timings of the two-stage cure cycle (4D design space) were constrained by the inequalities

$$
\left\{\begin{array}{l}
t_{-} \text {heat }_{1} \leq 75 \mathrm{~min} \\
t_{-} \text {hold }_{1} \leq 45 \mathrm{~min} \\
t_{-} \text {heat }_{2} \leq 90 \mathrm{~min} \\
t_{-} \text {hold }_{2} \leq 40 \mathrm{~min} \\
\sum t_{i} \leq 240 \mathrm{~min}
\end{array}\right.
$$

Full cycle duration was limited by the value 240 min due to manufacturing conditions.

Conversion at the end of cure cycle was calculated by integration and averaging over the cured composite volume $\Omega$; it was assumed to be no less than 0.98

$$
\langle\alpha\rangle=\int_{\Omega} \alpha\left(\mathbf{r}, t_{\text {end }}\right) \cdot d v / V \leq 0.98
$$

Cure process objectives included two variables that should be minimized. First objective is the maximum of the mean deviation of conversion during the cure cycle

$$
\langle\Delta \alpha\rangle=\max _{t \in\left[0 ; t_{\text {end }}\right]} \int_{\Omega} \mid \alpha(\mathbf{r}, t)-\langle\alpha\rangle \cdot d v / V .
$$

The second objective is the mean deviation of the temperature at the first and second stages of heating-up. This objective, which allows to estimate the temperature uniformity within the cured prepreg, was calculated according to the following relationships

$$
\langle\Delta T\rangle_{1,2}=\max _{t \in\left\{\left[0 ; t_{1}\right]\right.} \int_{\substack{\left[t_{2} ; t_{3}\right] \\ \Omega^{\Omega}}}\left|T(\mathbf{r}, t)-\langle T(t)\rangle_{1,2}\right| \cdot d v / V,
$$

where mean temperature at the heating stages of cure cycle

$$
\langle T(t)\rangle_{1,2}=\int_{\Omega} T(\mathbf{r}, t) \cdot d v / V ; \quad \text { at } \quad t \in\left\{\begin{array}{l}
{\left[0 ; t_{1}\right]} \\
{\left[t_{2} ; t_{3}\right]}
\end{array} .\right.
$$

These values of both objectives calculated at the different control parameters are the points in the objective space that were analyzed to find the reasonable minimum of criteria (9) and (10). Most attractive cases can be analyzed also not only for averaged, but local distribution of both objectives.

A multidimensional visualization techniques used here transforms a multidimensional problem so that it can be mapped to a $2 \mathrm{D}$ visual space. In the considered case when a control law is parameterized by four parameters whose values completely characterize the thermal action on the cure system, techniques based on multiple 2D displays is the most appropriate for providing insight into complex cure phenomena. We use some projections of the subset in the objective space to the $2 \mathrm{D}$ design subspace where these projections are drawn as level lines.

\begin{tabular}{|c|c|c|c|c|c|}
\hline \multirow[b]{2}{*}{ Schedule } & \multirow[b]{2}{*}{ Marker } & \multicolumn{4}{|c|}{ Timings, min (see Fig.1) } \\
\hline & & $\begin{array}{c}\mathrm{t} \\
\text { heat }\end{array}$ & hold ${ }_{1}$ & $\begin{array}{c}\mathrm{t}_{-} \\
\text {heat } \\
2\end{array}$ & ${ }_{\text {hold }}{ }_{2}$ \\
\hline $1^{\text {st }}$ & & 70 & 42 & 90 & 35 \\
\hline $2^{\text {nd }}$ & & 64 & 36 & 82 & 35 \\
\hline
\end{tabular}

Some optimization results are present in Figs. 10a and 10b. Four and six-pointed stars denote the temperature control laws, parameters of which are present in Table 2.

Table 2. Design variables for the studied control schedules.

The plots in Fig. 10 demonstrate that durations of first heating and first isothermal hold have the greatest impact on the spatial uniformity of degree of cure. It can be explained by the fact that these timings provide a uniform spatial distribution of $\alpha$ directly before the start of the second heating, when cross-linking reaction evolves very fast and this reaction is controlled not only by the temperature, but by time too. This is due to the increased influence of the multiplier $\alpha^{m}$ at the end of the first isothermal hold (see Eq. 2). The highest variation in the degree of cure occurs at the initial period of the second heating stage as resin gelation begins as shown in Figure 12. The highest cure quality can be attained through the acceptable deceleration of the heating rate. Figures 11 and 12 demonstrate time histories for the temperature in autoclave; averaged temperature and degree of cure deviations for two studied control schedules (see Table 2). It should be noted that the correct quantitative conclusions can only be reached when taking into account the actual geometry of the composite structure and the mould as well as their material properties. This confirms the need for use CAD model of cured parts. 


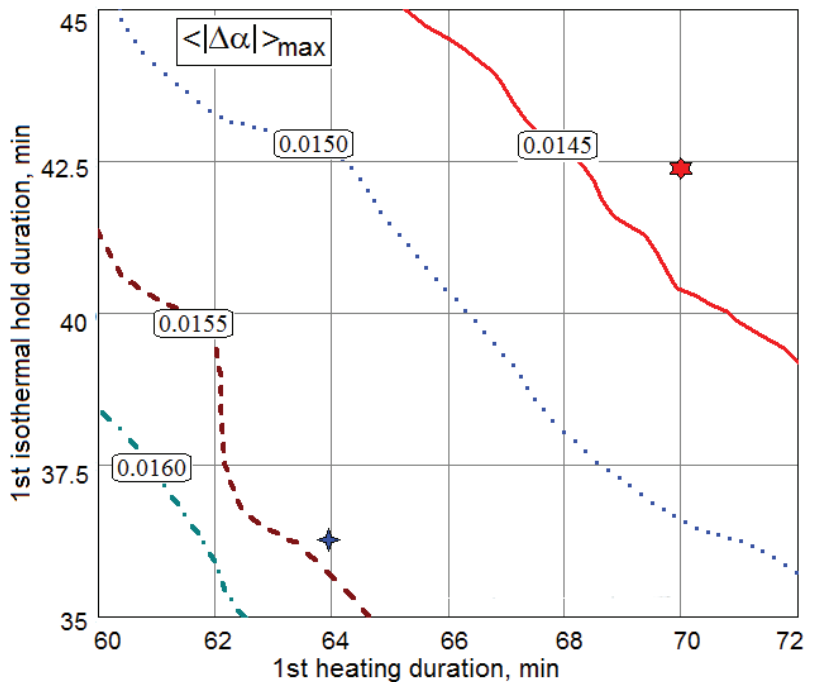

a)

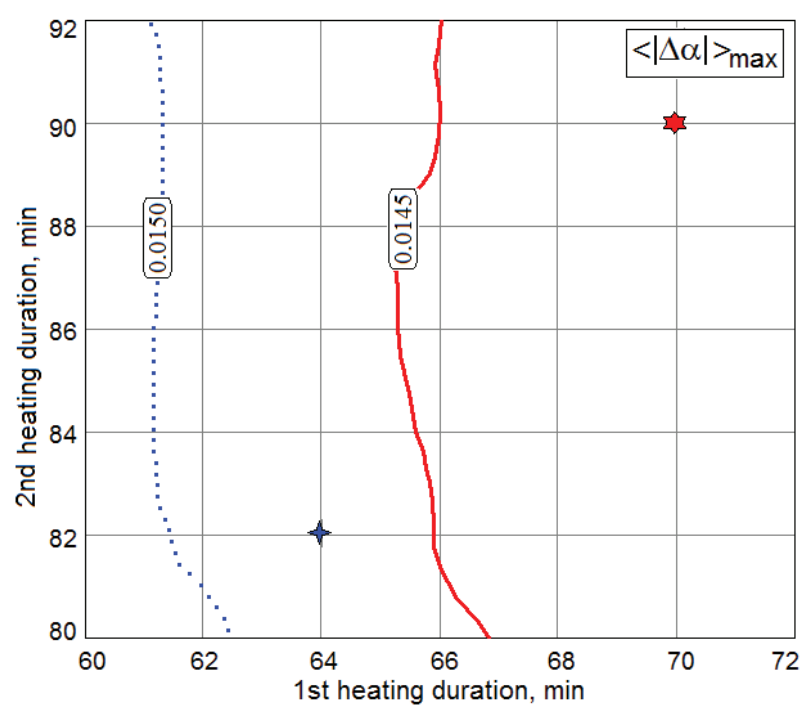

b)

Figure 10. Contour lines for the maximum variation of the degree of cure within the prepreg body througout the cycle.

a) Projection on the plane ( $t$ heat $t_{1}, t_{-}$hold $\left._{1}\right)$;

b) projection on the plane ( $t_{-}$heat $_{1}, t_{-}$heat $\left._{2}\right)$

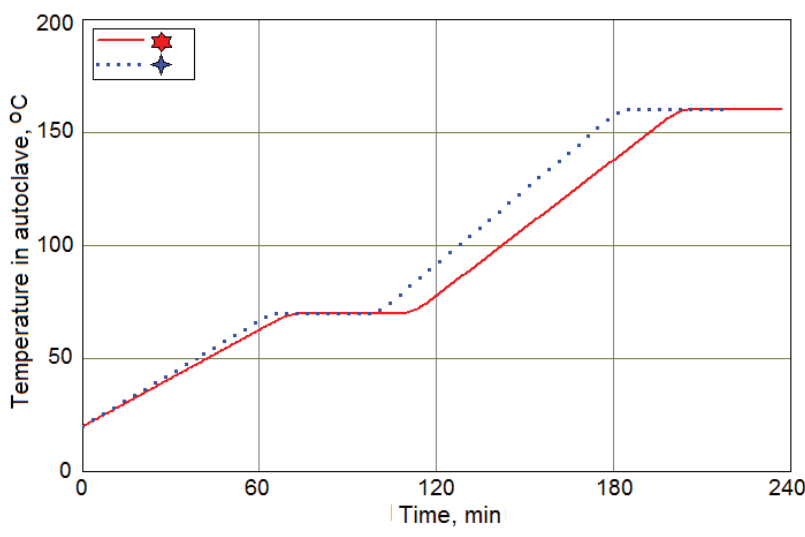

Figure 11. Two time histories of the temperature in atoclave

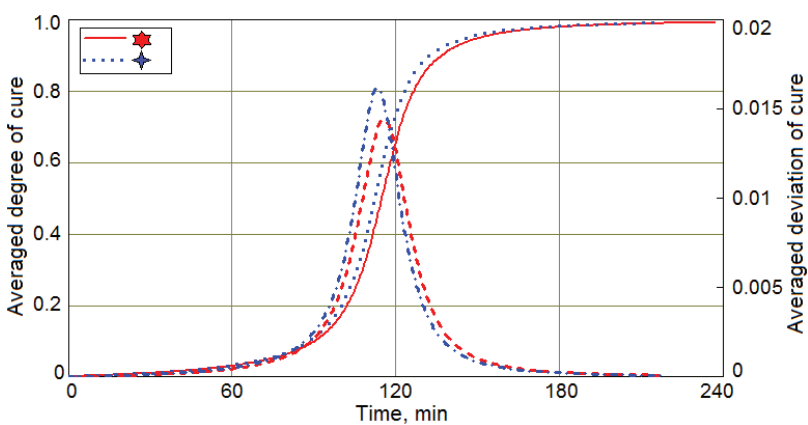

a)

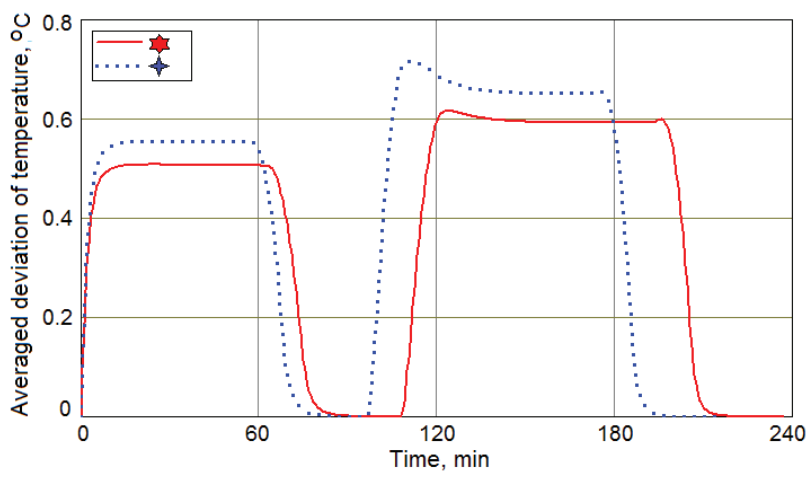

b)

Figure 12. The time histories of degree of cure $\alpha(t)$, its deviation (a) and deviation of temperature (b) averaged over prepreg volume at the curing schedules, which are present in Figure 11 (see Table 2)

\section{Conclusions}

We have defined and solved the problem of the optimal control synthesis for the cure process of large CFRP composite panel cured under vacuum in an autoclave as a multi-objective optimization problem to ensure minimum variations of the degree of cure and temperature inside the prepreg body throughout the cure cycle, taking into account all constraints imposed by the prepreg thermalkinetic properties and other operating conditions. The forward modeling problem for the transient cure process of the composite structure includes coupled heat transfer and kinetic phenomena in the prepreg throughout the two-stage cure cycle using the part geometry, which is exported from the CAD model to the finite element soft package. The input parameters of the process model implemented by using Comsol Multiphysics software are timings that form the control law for the temperature in an autoclave and appear as points in the 4D design space. The process quality criteria computed by integration over the cured prepreg volume at each time step are the averaged deviations of degree of cure and temperature determined at the completion of the transient analysis covering the entire cure cycle time. The suggested approach allows understanding the complex nature of curing phenomena, 
measuring the maximum achievable process quality, finding values satisfying the constraints imposed by the control law parameters, and making a reasonable decision on the choice of such values.

\section{References}

1. A. Baker, S. Dutton and D. Kelly, Composite Materials for Aircraft Structures, 2nd ed., (AIAA Itd., Reston, Virginia, 2004)

2. P.K. Mallick, Fiber-Reinforced Composites: Materials, Manufacturing, and Design, 3rd ed. (CRC Press, Boca Raton, FL, 2007)

3. G. Chiesura et al. Sensors. 16, 6 (2016)

4. H.C. Park, and S.W. Lee, J. Compos. Mater. $\mathbf{3 5}$ (2001)

5. Y. Gu, M. Li, Z. Zhang and Z. Sun, J. Compos. Mater. 40 (2006)

6. T.W. Capehart, and H.G. Kia, J. Compos. Mater. 41 (2007)

7. L. Sorrentino, W. Polini and C. Bellini, Adv. Compos. Mater. 23, 3, (2014)

8. S. Johnson, C.D. Rudd and D.J. Hill, P. I. Mech. Eng. B-J. Eng. 209 (1995)

9. J.-H. Oh and D.-G. Lee, J. Compos. Mater. 36, 1 (2002)

10. A. Mamoune, A. Saouab, T. Ouahbi and C.-H. Park, J. Reinf. Plast. Comp. 30 (2011)

11. P.E. Jahromi, A. Shojaei and S.M.R. Pishvaie, J. Reinf. Plast. Comp. 31 (2012)

12. A. Saad, A. Echchelh, M.Hattabi and M. El Ganaoui, J. Reinf. Plast. Comp. 31 (2012)

13. J. Xu, M. Qiao, B. You, and Y. Yu, J. Reinf. Plast. Comp. 31 (2012)

14. L. Sorrentino, C. Bellini, Polym.-Plast. Technol. 54 (2015)

15. I.V. Tarasov, S.N. Shevtsov, A.V. Evlanov and E.E. Orozaliev, IFAC P. Ser. 28, 11 (2016)

16. R.B. Williams, B.W.Grimsley, D.J. Inman, and W.K. Wilkie, J. Reinf. Plast. Comp. 23 (2004)

17. L. Esposito, L. Sorrentino, F. Penta, C. Bellini, Int. J. Adv. Manuf. Tech. 83, 5-8 (2016)

18. C. Brauner, T. French and A.S. Hermann, J. Compos. Mater. 51, 7 (2017)

19. J.-K. Wu et al. Proc. Int. Conf. on Information Science, Electronics and Electrical Engineering ISEEE 2014. (2014)

20. R.V.N. Melnik, Model. Simul. Mater. Sc. 10 (2002)

21. M.R. Kamal, S. Sourour, Polym. Eng. Sci. 13 (1973)

22. M.-K. Um, I.M. Daniel and C.-S. Hwang, Compos. Sci. Technol. 62 (2002)

23. J. Kim, T.J. Moon, and J.R. Howell, J. Compos. Mater. 36, 21 (2002)

24. B.-C. Chern, T.J. Moon, J.R. Howell and W. Tan, J. Compos. Mater. 36 (2002)

25. Wen, X., et al. High Perform. Polym. 23 (2011)
26. M.J. Yoo, et al. Eur. Polym. J. 46 (2010)

27. J. Zhang et al. Thermochim. Acta. 549 ( 2012)

28. R. Hardis, J.L.P. Jessop, F.E. Peters and M.R. Kessler, Compos. Part A-Appl. S. 49 (2013)

29. G. Van Assche, S. Awier, and B. Van Mele, Thermochim. Acta 388 (2002)

30. M. Vafayan, M.H. Beheshty, M.H.R. Ghoreishy and H. Abedini, J. Compos. Mater. 48 (2014) 